\title{
Changes in pulmonary function and patient-reported outcomes during COVID-19 recovery: a longitudinal, prospective cohort study
}

\author{
Aditi S. Shah $\mathbb{1}^{1}$, Min Hyung Ryu $\mathbb{B}^{1}$, Cameron J. Hague ${ }^{2}$, Darra T. Murphy $\mathbb{( D}^{2}$, James C. Johnston $\mathbb{B}^{3}$, \\ Christopher J. Ryerson $\mathbb{D}^{4}$, Christopher Carlsten $\mathbb{( D D}^{1,5}$ and Alyson W. Wong ${ }^{4,5}$
}

\begin{abstract}
${ }^{1}$ Division of Respiratory Medicine, Dept of Medicine, University of British Columbia, Vancouver General Hospital, Vancouver, Canada. ${ }^{2}$ Dept of Radiology, University of British Columbia, St Paul's Hospital, Vancouver, Canada. ${ }^{3}$ Division of Respiratory Medicine, Dept of Medicine, University of British Columbia, BC Centre for Disease Control, Vancouver, Canada. ${ }^{4}$ Division of Respiratory Medicine, Dept of Medicine, University of British Columbia, Centre for Heart Lung Innovation, St Paul's Hospital, Vancouver, Canada. ${ }^{5}$ These authors contributed equally.
\end{abstract}

Corresponding author: Christopher Carlsten (carlsten@mail.ubc.ca)

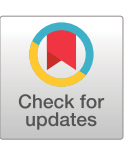

Copyright $\odot$ The authors 2021

This version is distributed under the terms of the Creative Commons Attribution Non-Commercial Licence 4.0. For commercial reproduction rights and permissions contact permissions@ersnet.org

This article has supplementary material available from openres.ersjournals.com

Received: 7 April 2021

Accepted: 11 June 2021

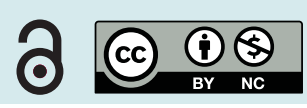

Shareable abstract (@ERSpublications)

\#COVID19 survivors have improvement in pulmonary function at 6 months. However, 83\% have abnormal patient-reported outcomes with $42 \%$ reporting persistent dyspnoea despite some with normal $D_{\text {LCO }}$ Imaging features at 3 months can help predict $D_{\text {Lco }}$ trajectory. https://bit.ly/3gEErST

Cite this article as: Shah AS, Ryu MH, Hague CJ, et al. Changes in pulmonary function and patientreported outcomes during COVID-19 recovery: a longitudinal, prospective cohort study. ERJ Open Res 2021; 7: 00243-2021 [DOI: 10.1183/23120541.00243-2021].

\section{Abstract}

Objectives The aim of this study was to compare respiratory and patient-reported outcome measures (PROMs) between 3 and 6 months after symptom onset and to identify features that predict these changes. Methods This was a consecutive prospective cohort of 73 patients who were hospitalised with coronavirus disease 2019 (COVID-19). We evaluated the changes in pulmonary function tests and PROMs between 3 and 6 months and then investigated the associations between outcomes (change in diffusing capacity of the lung for carbon monoxide $\left(D_{\mathrm{LCO}}\right)$, dyspnoea and quality of life $(\mathrm{QoL})$ ) and clinical and radiological features. Results There was improvement in forced vital capacity, total lung capacity and $D_{\text {Lco }}$ between 3 and 6 months by $3.25 \%, 3.82 \%$ and $5.69 \%$, respectively; however, there was no difference in PROMs. Reticulation and total computed tomography (CT) scores were associated with lower $D_{\text {LCO }} \%$ predicted at 6 months (coefficients; -8.7 and -5.3 , respectively). The association between radiological scores and $D_{\text {LCO }}$ were modified by time, with the degree of association between ground glass and $D_{\text {LCo }}$ having decreased markedly over time. There was no association between other predictors and change in dyspnoea or QoL over time.

Conclusions There is improvement in pulmonary function measurements between 3 and 6 months after COVID-19 symptom onset; however, PROMs did not improve. A higher reticulation and total CT score are negatively associated with $D_{\text {LCO }}$, but this association is attenuated over time. Lastly, there is a considerable proportion of patients with unexplained dyspnoea at 6 months, motivating further research to identify the underlying mechanisms.

\section{Introduction}

The coronavirus disease 2019 (COVID-19) pandemic has resulted in over 2 million deaths globally as of April 2021 [1]. Several follow-up studies have described abnormalities in patient-reported outcome measures (PROMs), pulmonary function tests (PFTs) and chest imaging months after COVID-19 [2-6]. However, it is unclear how these short-term abnormalities change over time and whether long-term patient outcomes can be predicted.

We have previously demonstrated that $50 \%$ of patients had dyspnoea and impairments in quality of life (QoL) 3 months after symptom onset and that a striking proportion of patients (88\%) had abnormalities on 
imaging, in particular ground glass opacities and reticulation [6]. Similar to other studies, we also showed that the diffusing capacity of the lung for carbon monoxide $\left(D_{\mathrm{LCO}}\right)$ was the most frequently impaired pulmonary function measurement among COVID-19 survivors [4, 5]. A disturbing feature of COVID-19 has been the identification of a subgroup of patients whose symptoms persist months after initial symptom onset and are seemingly out of proportion to what would be expected based on common investigations (e.g. unexplained dyspnoea). In addition to suffering with these abnormalities, patients are burdened by not knowing whether these sequelae will improve. Therefore, being able to understand how outcomes change over time would help inform discussions between clinicians and patients.

In this study, we sought to determine how respiratory symptoms, QoL and PFTs change over time during COVID-19 recovery and to identify features that predict these changes. We hypothesised that a higher burden of ground glass and/or reticulation on imaging and the presence of unexplained dyspnoea at 3 months post-COVID-19 symptom onset would be associated with greater improvement in respiratory and patient-reported outcomes at 6 months.

\section{Methods}

\section{Study population}

This study included a consecutively enrolled prospective cohort of patients hospitalised with COVID-19 in Vancouver, Canada between March and June 2020. Hospitalisation rates in this population are 5\% of all patients who tested positive for severe acute respiratory syndrome coronavirus 2 (SARS-CoV-2), of whom $20 \%$ required intensive care unit admission (i.e. 1\% of all SARS-CoV-2-positive patients) [7]. At discharge, patients admitted to hospital were automatically referred to the Post-COVID-19 Respiratory Clinic (PCRC), which is located at two academic hospitals. Patients were eligible for enrolment if they were hospitalised for COVID-19 (confirmed by positive SARS-CoV-2 PCR), were able to complete study questionnaires in English and were $\geqslant 18$ years of age. There were no exclusion criteria. All patients provided informed written consent (UBC Clinical Research Ethics Board \#H20-01239). The 3-month respiratory outcomes and PROMs for this cohort have been previously reported [5, 6].

\section{Measurements}

Clinical data were obtained from patient surveys and chart reviews. PFTs were conducted in accordance with international guidelines [8-11]. Transthoracic echocardiogram (TTE) was performed according to established guidelines and interpreted by cardiologists with advanced echocardiography training [12, 13]. All measurements were collected at both the 3- and 6-month visits (timed from COVID-19 symptom onset), except for the high-resolution computed tomography (HRCT) of the chest and TTE, which were only obtained at 3 months. Time from symptom onset was treated as a categorical variable (i.e. 3 and 6 months).

Two fellowship-trained cardiothoracic radiologists with 12 and 14 years of experience (DM, CH) independently scored the HRCTs of the chest. The HRCT ground glass and reticulation scores were determined using a standardised approach. The lungs were divided into six zones, and the extent of ground glass and reticulation were scored as a per cent of affected lung volume for each zone. The mean of these zones was then used to determine the overall HRCT ground glass and reticulation scores. The total HRCT score was the sum of the overall ground glass and reticulation scores $[14,15]$. The means of the scores from the two radiologists were used for the study. A $10 \%$ threshold was used for the abnormal percentage of lung involvement. This value is double the threshold (5\%) used to define interstitial lung abnormalities, which are mild interstitial abnormalities in people who have not been diagnosed with an interstitial lung disease [16]. Intraclass correlation coefficient (ICC) was used to determine interobserver agreement between the two radiologists.

PROMs were assessed using standardised questionnaires completed by patients [17]. Participants completed the following validated questionnaires at each study visit: University of California San Diego Shortness of Breath Questionnaire (UCSD), Cough Visual Analogue Scale (Cough VAS), Patient Health Questionnaire-9 (PHQ-9), Pittsburgh Sleep Quality Index (PSQI) and EuroQol- 5 Dimension (EQ-5D) [18-24]. PSQI and PHQ-9 provide assessment of sleep and depression, respectively. A global PSQI score $>5$ indicates poor sleep and PHQ-9 $\geqslant 5$ suggests the presence of a mood disorder. Cough VAS was utilised for cough assessment [22, 23]. A value $\geqslant 17 \mathrm{~mm}$ was considered abnormal [24]. The EQ-5D is a generic preference-based instrument that measures QoL in five dimensions. The EQ-5D score is then converted to a health utility index, which typically ranges between 0 to 1 , with 1 representing perfect health and 0 representing death. The EQ-5D also includes a visual analogue scale (VAS), where patients rate their current health from 0 to 100, with a higher value indicating better health [19]. The health utility index and EQ-5D VAS values were compared to the mean Canadian population norm, which is 0.931 and 77.1, 
respectively, for people in the 65 to 74 years age group [19]. The UCSD questionnaire was used to grade severity of dyspnoea and ranges from 0 to 120 [21]. Dyspnoea is considered present when the UCSD is $>5$, with a higher score representing worse dyspnoea [25]. To illustrate clinically meaningful dyspnoea, a UCSD score $>10$ was used to define the presence of dyspnoea.

Previous studies have described correlations between physiological parameters and dyspnoea [21]. For example, in patients with COVID-19, dyspnoea and $D_{\text {LCO }} \%$ predicted were negatively correlated [26]. Based on this, we defined unexplained dyspnoea as the presence of dyspnoea (UCSD dyspnoea score $>10$ ) in an individual with a normal $D_{\text {LCO }}(\geqslant 80 \%$ predicted).

\section{Outcomes}

The primary outcome was the change in $D_{\text {LCO }} \%$ predicted between 3 and 6 months after COVID-19 symptom onset, while the secondary outcomes were change in QoL (EQ-5D utility) and dyspnoea (UCSD) at these same times. These outcomes were predetermined based on our previous studies demonstrating that these variables were frequently abnormal 3 months after symptom onset $[5,6]$.

\section{Statistical analyses}

We constructed seven models to address our prespecified hypotheses and support risk prediction during COVID-19 recovery. Models 1a-c tested the association between ground glass and/or reticulation scores with change in $D_{\text {LCO }}$ over time, with a goal to determine whether imaging at 3 months would predict subsequent change in physiological parameters at 6 months. Models 2a and 2b investigated the association between ground glass at 3 months with change in dyspnoea (UCSD score) and QoL (EQ-5D utility) from 3 to 6 months. Models 3a-b tested association between the presence of unexplained dyspnoea at 3 months with changes in dyspnoea and QoL from 3 to 6 months.

Linear mixed effects models were used to explore changes in outcomes over time and were adjusted for age, sex and smoking pack-years. The models included a random intercept and random slope to account for the variability between patients that is not accounted for by the included covariates. Interaction terms were included, since the primary predictor variables may have a different effect on the outcome depending on time from symptom onset.

Normally distributed continuous variables were expressed as means \pm sD; non-normally distributed variables were expressed as median (interquartile range). A paired t-test or Wilcoxon signed rank test was used to compare measurements between 3 and 6 months. A two-sided p-value $<0.05$ was considered statistically significant. Statistical analyses were performed using SPSS (version 22.0; SPSS, Chicago, IL, USA) and R (version 3.6.3).

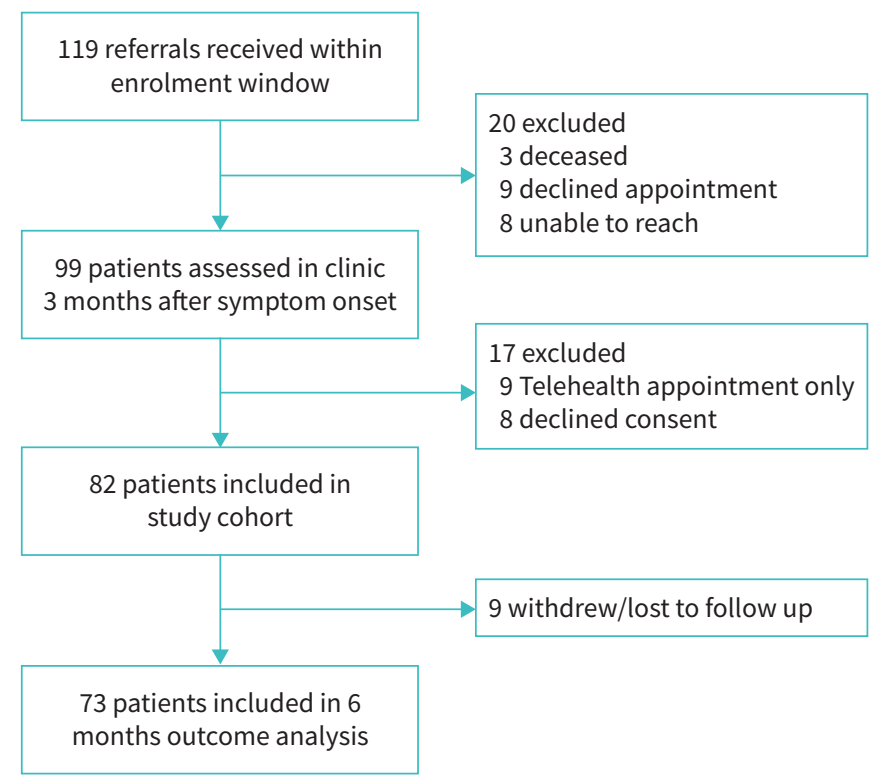

FIGURE 1 Flow diagram of study enrolment. 
Results

Baseline characteristics

A total of 73 patients admitted between March and June 2020 were included in this study as outlined in figure 1. The demographics and baseline characteristics are displayed in table 1 . The median duration from symptom onset was 13 weeks (IQR 11-14) and 27 weeks (IQR 24-30) for the 3- and 6-month visits, respectively. The median age was 65 years (IQR 53-72). The majority of the cohort were male (60\%) and approximately one-third had a history of smoking (32\%). There were $64 \%$ of patients with dyspnoea (UCSD dyspnoea score $>5$ ) and 42\% with more severe dyspnoea (UCSD dyspnoea score $>10$ ). TTE was performed in 72 patients at the 3-month visit; median left ventricular (LV) systolic function (LV ejection fraction 60\% (IQR 60-65)) and median estimated pulmonary artery systolic pressure (27 mmHg (IQR 2330)) were normal.

TABLE 1 Clinical characteristics and pulmonary function tests of patients hospitalised with coronavirus disease 2019 (COVID-19), 6 months after symptom onset

\begin{tabular}{|c|c|c|c|c|}
\hline \multirow[t]{2}{*}{ Features } & \multirow[t]{2}{*}{ Overall cohort } & \multicolumn{2}{|c|}{ Patients with dyspnoea at 6 months } & \multirow[t]{2}{*}{ p-value } \\
\hline & & Unexplained dyspnoea & Dyspnoea & \\
\hline Subjects $n$ & 73 & 13 & 15 & \\
\hline \multicolumn{5}{|l|}{ Demographics } \\
\hline Age years & $65(53-72)$ & $49(34-67)$ & $66(59-76)$ & 0.02 \\
\hline Male sex $n(\%)$ & $44(60)$ & $4(31)$ & $9(60)$ & 0.12 \\
\hline Ever-smoker n (\%) & $23(32)$ & $2(15)$ & $8(53)$ & 0.06 \\
\hline \multicolumn{5}{|l|}{ Comorbidities n (\%) } \\
\hline Hypertension & $27(37)$ & $5(39)$ & $8(53)$ & 0.43 \\
\hline Diabetes & $19(26)$ & $3(23)$ & $5(33)$ & 0.69 \\
\hline Chronic pulmonary disease $\mathrm{A}^{\#}$ & $10(14)$ & 0 & $4(27)$ & 0.10 \\
\hline Coronary heart disease & $7(10)$ & 0 & $3(20)$ & 0.23 \\
\hline Malignancy & $8(11)$ & $1(8)$ & $1(7)$ & 1.00 \\
\hline Chronic kidney disease & $6(8)$ & $1(8)$ & $2(13)$ & 1.00 \\
\hline \multicolumn{5}{|l|}{ Respiratory symptoms } \\
\hline UCSD dyspnoea score & $9(3-31)$ & $31(17-40)$ & $35(23-46)$ & 0.27 \\
\hline Cough VAS mm & $20(10-37)$ & $10(9-10)$ & $30(16-44)$ & 0.07 \\
\hline \multicolumn{5}{|c|}{ Patient-reported outcome measures } \\
\hline EQ-5D health utility & $0.9(0.8-0.9)$ & $0.83(0.77-0.87)$ & $0.83(0.76-0.87)$ & 0.79 \\
\hline EQ-5D VAS & $80(75-90)$ & $75(70-90)$ & $75(65-85)$ & 0.50 \\
\hline PSQI & $\begin{array}{l}5(2-9) \\
(\mathrm{n}=72)\end{array}$ & $\begin{array}{c}9(6-12) \\
(n=12)\end{array}$ & $7(5-9)$ & 0.28 \\
\hline PHQ-9 & $1(0-6)$ & $6(2-10)$ & $5(1-7)$ & 0.39 \\
\hline \multicolumn{5}{|l|}{ Pulmonary function tests } \\
\hline $\mathrm{FEV}_{1} \%$ predicted & $\begin{array}{l}91 \pm 15 \\
(n=72)\end{array}$ & $88 \pm 14$ & $83 \pm 14$ & 0.31 \\
\hline FVC \% predicted & $\begin{array}{l}93 \pm 16 \\
(n=72)\end{array}$ & $93 \pm 11$ & $81 \pm 15$ & 0.03 \\
\hline $\mathrm{FEV}_{1} / \mathrm{FVC} \%$ & $\begin{array}{l}84 \pm 12 \\
(n=72)\end{array}$ & $84 \pm 13$ & $84 \pm 11$ & 0.88 \\
\hline TLC \% predicted & $\begin{array}{l}87 \pm 13 \\
(n=64)\end{array}$ & $\begin{array}{l}86 \pm 11 \\
(n=12)\end{array}$ & $\begin{array}{l}77 \pm 13 \\
(n=14)\end{array}$ & 0.09 \\
\hline$D_{\text {LCo }} \%$ predicted & $\begin{array}{l}79 \pm 18 \\
(n=70)\end{array}$ & $88 \pm 9$ & $63 \pm 14$ & $<0.001$ \\
\hline \multicolumn{5}{|c|}{ Transthoracic echocardiogram $(n=72)$} \\
\hline LVEF \% & $60(60-65)$ & $60(60-64)$ & $65(60-65)$ & 0.29 \\
\hline PASP mmHg & $27(23-30)$ & $19(23-34)$ & $27(23-30)$ & 0.77 \\
\hline \multicolumn{5}{|c|}{ 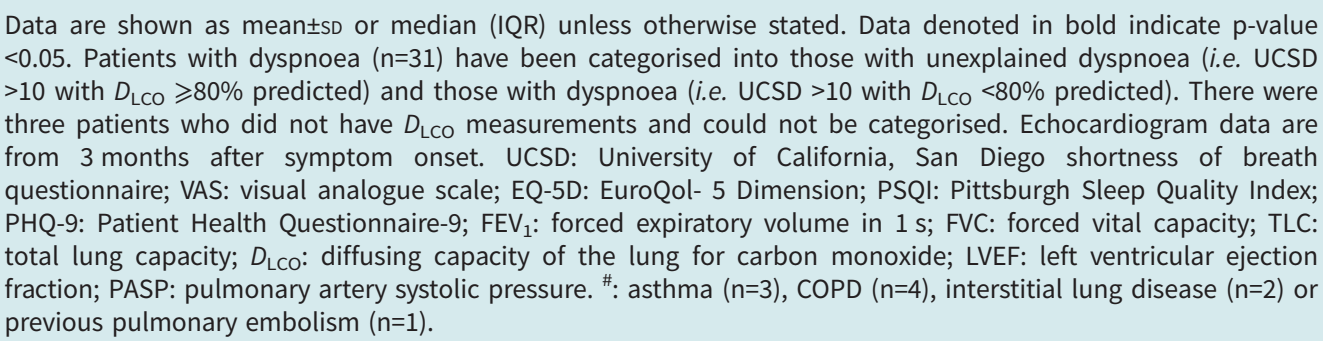 } \\
\hline
\end{tabular}


Unexplained dyspnoea (i.e. UCSD score $>10$ and $D_{\mathrm{LCO}} \geqslant 80 \%$ predicted) was present in a similar proportion of patients at 3 and 6 months (14\% and 19\%), with median UCSD scores of 25 (IQR 14-26) and 31 (IQR 17-40), respectively (table 1). Among patients with unexplained dyspnoea, reduced QoL was present in $70 \%$ and $92 \%$ of patients at 3 and 6 months, respectively. The most common abnormal PROMs were reduced QoL (92\%) and poor sleep (69\%). Characteristics of patients with and without dyspnoea (UCSD >10), irrespective of $D_{\mathrm{LCO}}$, are provided in supplementary table S1. Patients with dyspnoea had greater impairments in physiological measurements and PROMs compared to patients without dyspnoea.

The median percentage of lung affected by ground glass and reticulation on 3-month HRCT was $<10 \%$ in these patients. There was excellent interobserver agreement between the two radiologists with ICCs of 93\%, $75 \%$ and $87 \%$ for ground glass, reticulation and total computed tomography (CT) score respectively.

\section{Change in pulmonary function over time}

Changes in pulmonary outcomes from 3 to 6 months after symptom onset are shown in table 2 and figure 2. There was improvement in forced expiratory volume in $1 \mathrm{~s}$ (FVC) (mean difference, 3.25\%; 95\% CI: 1.31-5.19; $\mathrm{p}=0.001$ ), TLC (mean difference, 3.82\%; 95\% CI: 2.16-5.49; $\mathrm{p}<0.001$ ) and $D_{\text {LCO }}$ (mean difference, 5.69\%; 95\% CI: 3.56-7.82; $<<0.001$ ) between 3 and 6 months. There was a significant decline in $\mathrm{FEV}_{1} / \mathrm{FVC}$ ratio (mean difference, $-2.86 \%$; 95\% CI: -4.45 to -1.26 ; $\mathrm{p}=0.001$ ), which was consistent with less ventilatory restriction over time. The proportion of patients with an abnormal $D_{\text {LCO }}(<80 \%$ predicted) decreased from $59 \%$ at 3 months to $46 \%$ at 6 months $(\mathrm{p}<0.001)$. There was no difference in the proportion of patients requiring mechanical ventilation during their acute COVID-19 illness between those with normal versus abnormal $D_{\mathrm{LCO}}$ at 6 months (21\% and 25\%, respectively).

\section{Change in PROMs over time}

The change in PROMs from 3 to 6 months after symptom onset is shown in table 2 and figure 2. At 6 months, 84\% of patients had at least one abnormal PROM. QoL was the most common abnormality at 6 months, with $70 \%$ of the cohort having an EQ-5D utility (preference value that patients attach to their overall health status) worse than the population norm [19, 27]. There was no change in QoL based on the EQ-5D utility; however, there was an improvement in median EQ-5D VAS from 3 to 6 months (median difference, 6.32; 95\% CI: 5.00-9.50, $\mathrm{p}<0.001$ ). There was no change in mood or sleep. Despite the improvement in PFT values, there was no significant change in median cough or dyspnoea scores, with at least one of these symptoms present in $45 \%$ of patients at 6 months.

TABLE 2 Respiratory symptoms, patient-reported outcome measures and pulmonary function at 3 and 6 months after coronavirus disease 2019 (COVID-19) symptom onset

\begin{tabular}{|c|c|c|c|c|c|}
\hline & 3 months & 6 months & $\begin{array}{l}\text { Mean or median } \\
\text { difference }\end{array}$ & $95 \% \mathrm{Cl}$ & $\mathrm{p}$-value \\
\hline \multicolumn{6}{|c|}{ Respiratory symptoms } \\
\hline $\begin{array}{l}\text { UCSD dyspnoea } \\
\text { score }\end{array}$ & $11(3-26)$ & $9(3-31)$ & -1.0 & $-4.0-2.0$ & 0.53 \\
\hline Cough VAS & $28(8-60)$ & $20(10-35)$ & -4.6 & $-18.7-8.4$ & 0.41 \\
\hline \multicolumn{6}{|c|}{ Patient-reported outcome measures } \\
\hline PHQ-9 & $2(1-6)$ & $1(0-6)$ & 0.5 & $0-1.5$ & 0.16 \\
\hline PSQI & $5(3-8)$ & $5(2-9)$ & 0 & $-1.0-1.5$ & 0.81 \\
\hline EQ-5D utility & $0.87(0.79-0.95)$ & $0.90(0.81-0.95)$ & -0.022 & $-0.1-0.003$ & 0.12 \\
\hline EQ-5D VAS & $75(68-90)$ & $80(75-90)$ & 6.3 & $5.0-9.5$ & $<0.001$ \\
\hline \multicolumn{6}{|c|}{ Pulmonary function, \% predicted } \\
\hline $\mathrm{FEV}_{1}$ & $89 \pm 16$ & $91 \pm 16$ & 1.3 & $-0.8-3.4$ & 0.21 \\
\hline FVC & $90 \pm 17$ & $93 \pm 17$ & 3.3 & $1.3-5.2$ & 0.001 \\
\hline $\mathrm{FEV}_{1} / \mathrm{FVC}$ & $87 \pm 12$ & $84 \pm 12$ & -2.9 & $-4.5--1.3$ & 0.001 \\
\hline TLC & $83 \pm 14$ & $87 \pm 13$ & 3.8 & $2.2-5.5$ & $<0.001$ \\
\hline$D_{\mathrm{LCO}}$ & $74 \pm 17$ & $80 \pm 17$ & 5.7 & $3.6-7.8$ & $<0.001$ \\
\hline \multicolumn{6}{|c|}{$\begin{array}{l}\text { Data for } 3 \text { and } 6 \text { months are shown as mean } \pm \text { SD or median (IQR). Data denoted in bold indicate } \mathrm{p} \text {-value }<0.05 \text {. A } \\
\text { paired t-test or Wilcoxon signed rank test were used to compare values between } 3 \text { and } 6 \text { months. UCSD: } \\
\text { University of California, San Diego shortness of breath questionnaire; VAS: visual analogue scale; PHQ- } 9 \text { : Patient } \\
\text { Health Questionnaire-9; PSQI: Pittsburgh Sleep Quality Index; EQ-5D: EuroQol- } 5 \text { Dimension; FEV } V_{1} \text { : forced } \\
\text { expiratory volume in } 1 \mathrm{~s} \text {; FVC: forced vital capacity; TLC: total lung capacity; } D_{\text {Lco: }} \text { diffusing capacity of the lung } \\
\text { for carbon monoxide. }\end{array}$} \\
\hline
\end{tabular}



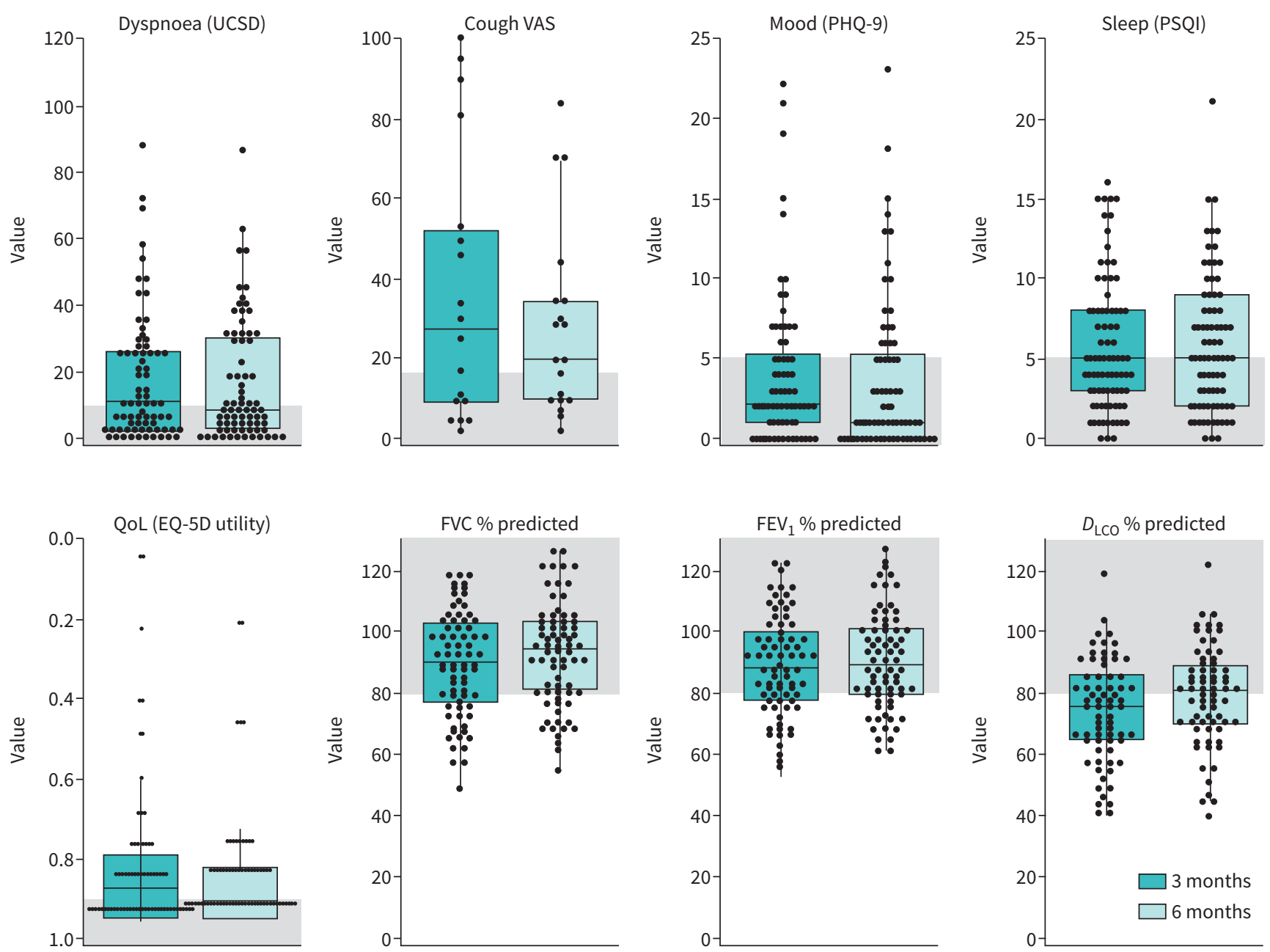

FIGURE 2 Patient-reported outcomes and pulmonary function measurements at 3 and 6 months after coronavirus disease 2019 (COVID-19) symptom onset. Each circle represents a patient, and the box represents the median and interquartile range. The $y$-axis shows the complete range of possible scores, and areas shaded in grey represent the normal range based on population adjusted norms where available. $D_{\mathrm{Lco}}$ : diffusing capacity of the lung for carbon monoxide; EQ-5D: EuroQol- 5 Dimension; FEV 1 : forced expiratory volume in 1 s; FVC: forced vital capacity; PHQ-9: Patient Health Questionnaire-9; PSQI: Pittsburgh Sleep Quality Index; QoL: quality of life; UCSD: University of California, San Diego shortness of breath questionnaire; VAS: visual analogue scale.

\section{Predictors of change \\ Change in PROMS}

There were 26 patients (36\%) with ground glass opacities involving $>10 \%$ of the lung on HRCT. Among these 26 patients, 9 (35\%) had dyspnoea and 18 (69\%) had an EQ-5D utility worse than the population norm at 6 months. The extent of ground glass present at 3 months was not associated with change in dyspnoea or QoL, and time did not significantly modify these associations (table 3, models 2a-b). Furthermore, there was no association between the presence of unexplained dyspnoea at 3 months and change in dyspnoea or QoL over time (table 3, models 3a-b).

\section{Change in $D_{\text {LCO }} \%$ predicted}

Reticulation and total HRCT score at 3 months were each associated with the change in $D_{\mathrm{LCO}}$ from 3 to 6 months (decrease of $8.7 \%$ predicted per unit increase in reticulation score; decrease of $5.3 \%$ predicted per unit increase in total HRCT score; table 3, models 1a-c). There was no association between 3-month ground glass and change in $D_{\mathrm{LCO}}$. However, time significantly modified the association between 3-month ground glass and $D_{\mathrm{LCO}}$ and also, with marginal significance, the association between 3-month reticulation and $D_{\mathrm{LCO}}(\mathrm{p}=0.05)$ and between 3-month total CT score and $D_{\mathrm{LCO}}(\mathrm{p}=0.07)$. The slope of the relationship between each of these initial radiographic abnormalities and $D_{\mathrm{LCO}}$ decreased from 3 months to 6 months (figure 3). 


\begin{tabular}{|c|c|c|c|c|c|c|}
\hline Model & Outcome & Predictor (at 3 months) & Coefficient & $95 \% \mathrm{Cl}$ & p-value & Prespecified covariates \\
\hline \multirow[t]{3}{*}{$1 a$} & $D_{\text {Lco }} \%$ predicted & Ground glass score & -2.2 & $-5.9-1.4$ & 0.23 & Sex, age, smoking pack-years \\
\hline & & Time & 0.8 & $-0.5-2.0$ & 0.21 & \\
\hline & & Ground glass score ${ }^{\star}$ Time & 0.6 & $0.05-1.2$ & 0.03 & \\
\hline \multirow[t]{3}{*}{$1 b$} & $D_{\text {Lco }} \%$ predicted & Reticulation score & -8.7 & $-12.1--5.4$ & $<0.001$ & Sex, age, smoking pack-years \\
\hline & & Time & 1.2 & $0.1-2.2$ & 0.03 & \\
\hline & & Reticulation score*Time & 0.6 & $-0.01-1.25$ & 0.05 & \\
\hline \multirow[t]{3}{*}{ 1c } & $D_{\text {Lco }} \%$ predicted & Total CT score & -5.3 & $-8.7--1.8$ & 0.003 & Sex, age, smoking pack-years \\
\hline & & Time & 0.8 & $-0.5-2.2$ & 0.23 & \\
\hline & & Total CT score ${ }^{\star}$ Time & 0.5 & $-0.04-1.05$ & 0.07 & \\
\hline \multirow[t]{3}{*}{$2 a$} & UCSD & Ground glass score & 0.8 & $-3.4-5.0$ & 0.69 & Sex, age, smoking pack-years \\
\hline & & Time & -0.2 & $-2.9-2.5$ & 0.88 & \\
\hline & & Ground glass score*Time & -0.1 & $-1.4-1.1$ & 0.87 & \\
\hline \multirow[t]{3}{*}{$2 b$} & QoL & Ground glass score & -0.003 & $-0.05-0.04$ & 0.89 & Sex, age, smoking pack-years \\
\hline & & Time & -0.003 & $-0.02-0.02$ & 0.77 & \\
\hline & & Ground glass score*Time & 0.01 & $-0.002-0.02$ & 0.15 & \\
\hline \multirow[t]{3}{*}{$3 a$} & UCSD & Unexplained dyspnoea & 5.16 & $-7.95-18.3$ & 0.43 & Sex, age, smoking pack-years \\
\hline & & Time & -0.45 & $-1.98-1.08$ & 0.56 & \\
\hline & & Unexplained dyspnoea*Time & 1.01 & $-2.98-5.01$ & 0.61 & \\
\hline \multirow[t]{3}{*}{$3 b$} & QoL & Unexplained dyspnoea & 0.01 & $-0.11-0.13$ & 0.88 & Sex, age, smoking pack-years \\
\hline & & Time & 0.01 & $-0.001-0.02$ & 0.07 & \\
\hline & & Unexplained Dyspnoea*Time & -0.01 & $-0.04-0.01$ & 0.33 & \\
\hline
\end{tabular}

Time was categorised as 3 months (reference) and 6 months from symptom onset. Ground glass, reticulation and total CT scores were continuous variables that were log-transformed to make them normally distributed and to meet model assumptions. Unexplained dyspnoea at 3 months (defined as the presence of a UCSD dyspnoea score $>10$ with $D_{\text {LCO }} \%$ predicted $\geqslant 80 \%$ ) was categorical (present or absent). Time was included as an interaction term to evaluate whether time modified the effect of the primary predictor on the outcome. The primary predictor variables are denoted in bold. Example of Model 1a interpretation: the coefficient of -2.2 for ground glass score indicates that each $1 \%$ increase in ground glass score is associated with a $2.2 \%$ decrease in $D_{\mathrm{LCO}} \%$ predicted. This association is modified by time. At 6 months, for each $1 \%$ increase in ground glass, the coefficient will increase by $1.4(0.8+0.6)$, which means there will be a $0.8 \%(-2.2+1.4)$ decrease in $D_{\text {Lco }} \%$ predicted at 6 months compared to a $2.2 \%$ decrease at 3 months. $D_{\text {LCo }}$ : diffusing capacity of the lung for carbon monoxide; UCSD: University of California, San Diego shortness of breath questionnaire; QoL: quality of life. Asterisks (*) signify interaction terms.

\section{Discussion}

This study demonstrates that there is improvement in most pulmonary function measurements between 3 and 6 months after symptom onset for patients hospitalised with COVID-19. However, improvement was less frequent for PROMs, with QoL (based on EQ-5D VAS) being the only PROM that significantly improved between 3 and 6 months. There was no significant change in dyspnoea or cough over time and nearly half of patients continued to experience these symptoms at 6 months. Importantly, 19\% of patients with dyspnoea at 6 months had normal $D_{\text {LCO }} \%$ predicted, highlighting the need to identify the underlying cause of this burdensome symptom that is frequently not explained by gas exchange abnormalities.

Our findings show that $D_{\text {LCO }}$ improves with time after COVID-19. However, 3-month CT scores, particularly the ground glass component, were less associated with $D_{\mathrm{LCO}}$ at the later assessment. Reticulation is indicative of pulmonary fibrosis that is not reversible and implies permanent physiological impairments, which may explain why the temporal change in association of $D_{\text {LCO }}$ with ground glass was greater than with reticulation. The presence of early ground glass thus appears to leave potential for reversible disease (physiological recovery over time). However, generalisability of this welcome finding is limited by the fact that the extent of ground glass was mild for the majority of patients in this cohort and that a 3-month time interval may be too short to see larger changes. Furthermore, it is not clear that ground glass is the only or predominant factor that influences $D_{\text {LCO }}$ trajectory in COVID-19 survivors. Previous studies have described the presence of distinct pulmonary vascular changes during acute COVID-19, which could impact change in $D_{\text {LCO }}$ over time $[28,29]$. That said, in our cohort, echocardiograms showed normal LV systolic function and estimated pulmonary artery systolic pressure. Longitudinal follow-up with blood biomarkers, further imaging including ventilation perfusion scans and PFTs will provide important insight into the possible correlation between pulmonary vascular sequelae and physiological outcomes after COVID-19.

Dyspnoea is one of the most common persistent symptoms experienced by patients during COVID-19 recovery, but its underlying cause remains elusive. In a longitudinal study with 2469 patients hospitalised 

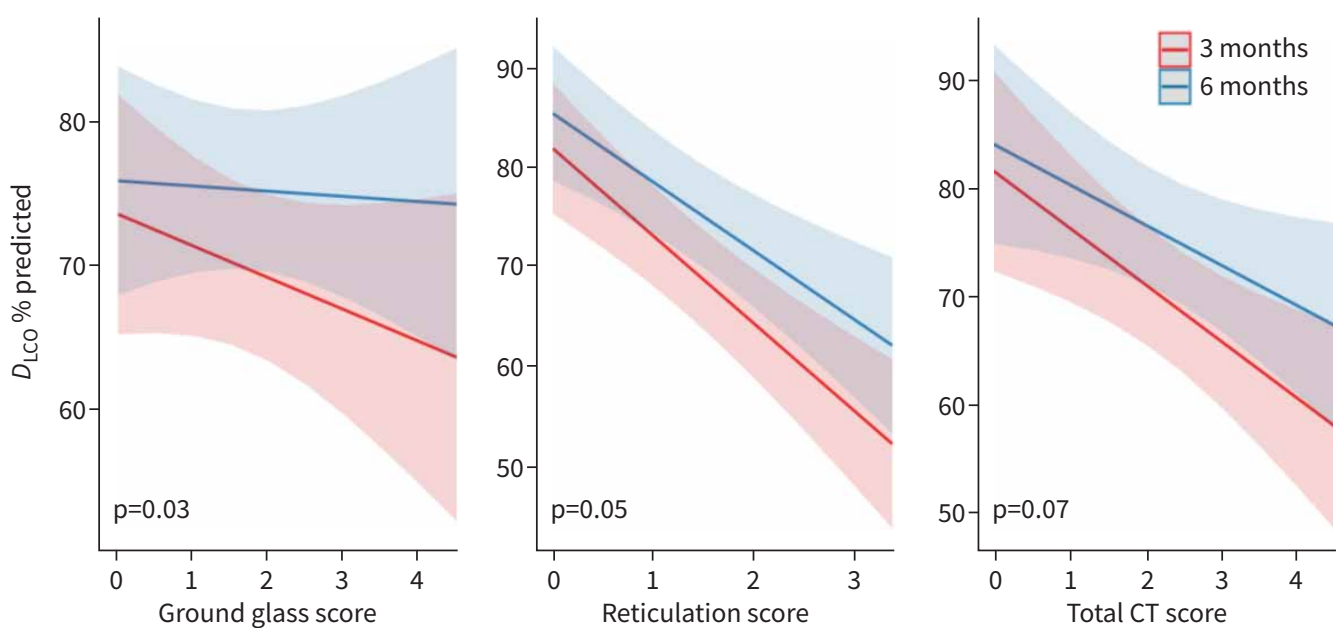

FIGURE 3 The effect of time on the association between radiological abnormalities and diffusing capacity of the lung for carbon monoxide $\left(D_{\text {LCO }}\right)$ at 3 and 6 months after coronavirus disease 2019 (COVID-19) symptom onset. The $x$-axis represents the log of each radiological score. This figure demonstrates the association between 3-month computed tomography (CT) scores (ground glass, reticulation and total CT scores) and $D_{\text {LCO }}$ $\%$ predicted. In each case, there is a negative relationship between the radiological abnormalities and $D_{\text {Lco }} \%$ predicted. However, this negative relationship is attenuated over time, as demonstrated by the shallower slopes at 6 months compared to 3 months.

with COVID-19, dyspnoea (modified Medical Research Council (mMRC) $\geqslant 1$ ) was present in $\sim 25 \%$ of patients 6 months after symptom onset [30]. In our study, the proportion of patients with clinically meaningful dyspnoea (UCSD >10) was $42 \%$ at 6 months. The difference in these proportions highlights the importance of careful selection of symptom questionnaires. The UCSD ranges between 0 and 120 which offers increased granularity when investigating severity or causes of dyspnoea compared to the mMRC which ranges between 0 and 4 [21, 31, 32]. The UCSD also has high test-retest reliability (i.e. consistently reproduces the same result over multiple visits when other variables remain the same), making it suitable for longitudinal studies [33].

There is growing awareness of a group of patients with persistent unexplained dyspnoea following COVID-19. In our cohort, unexplained dyspnoea was present in 14\% of the cohort at 3 months and $19 \%$ at 6 months. Our initial impression was that patients with unexplained dyspnoea would demonstrate improvement in dyspnoea and QoL over time given they may be more impaired at baseline with greater room for improvement. However, these associations were not seen in our study. Patients with unexplained dyspnoea represent a unique cohort with distinct outcomes. Further research into the respiratory and non-respiratory causes of unexplained dyspnoea and prognosis is greatly needed.

QoL (based on EQ-5D VAS) significantly improved with time and was comparable to the population norm by 6 months. This is a reassuring finding, as over $50 \%$ of patients reported abnormal QoL 3 months after symptom onset [6]. To our knowledge, there are no studies that have investigated predictors of QoL in COVID-19 survivors at 6 months. Huang and colleagues describe greater frequency of QoL impairments among patients warranting ventilation support during their acute illness; however, the association of QoL with imaging and clinical symptoms has not been investigated [30]. Our study shows that the severity of ground glass and presence of unexplained dyspnoea were not associated with change in QoL. However, QoL is not just determined by a person's health status but is also influenced by other important parameters such as psychosocial features and socioeconomic status. As such, our models may not have been comprehensive enough to identify predictors of change in QoL.

This study has several other limitations. Our sample size is modest, though our cohort has the advantage of being consecutively enrolled and well characterised, with standardised data collection and follow-up protocols all rigorously applied. Furthermore, our study is limited to a small proportion of patients who were hospitalised for COVID-19 and cannot be generalised to patients who were asymptomatic or treated as outpatients. Furthermore, the lack of PROMs and PFTs at baseline in these patients (preceding COVID-19) limits our ability to attribute these outcomes to COVID-19, although our longitudinal data 
partly address this. For example, if variables improved over time, then this would increase our confidence that the abnormalities were more likely attributable to recent COVID-19. Lastly, the definition of unexplained dyspnoea was synthesised for the purposes of this study and has not been validated. It remains possible that results could vary based on the instruments and the physiological and clinical variables (e.g. anaemia, deconditioning or cardiovascular comorbidities) used to characterise and define unexplained dyspnoea. In our cohort, only one patient had anaemia in the unexplained dyspnoea group, and their $D_{\text {LCO }}$ adjusted for haemoglobin remained $>80 \%$ predicted. There were also no differences in echocardiogram parameters between the groups, making cardiovascular abnormalities an unlikely source of unexplained dyspnoea. Further research is needed to better understand the cause of persistent symptoms after COVID-19.

\section{Conclusion}

The majority of patients discharged from hospital have residual symptoms, pulmonary function impairments and imaging abnormalities 3 months after COVID-19. Although physiological impairments improve over the subsequent 3 months, a similar improvement in patient-reported outcomes is not seen. Dyspnoea is one of the most persistent symptoms, despite a considerable proportion of these patients having normal PFTs. Further studies to determine the underlying causes and predictors of dyspnoea are needed in order to guide effective management for the staggering number of COVID-19 survivors worldwide.

Acknowledgements: The authors thank the patients who participated in this study. They also thank Agnes Yuen, Parteek Johal, Kevin Lau, Carley Schwartz and Kathryn Donohoe (all affiliated with Division of Respiratory Medicine, University of British Columbia) for their assistance with data entry and research support.

Provenance: Submitted article, peer reviewed.

Author contributions: A.W. Wong and C. Carlsten are co-senior authors and contributed equally to this work. A.S. Shah, A.W. Wong, C. Carlsten, C.J. Ryerson and J.C. Johnston contributed to study design, data analysis, data interpretation and writing of the manuscript. M.H. Ryu contributed to data analysis. C.J. Hague and D.T. Murphy provided chest CT interpretations, and contributed to data interpretation and writing of the manuscript. All authors read and approved the final version of the manuscript. All authors had full access to the data in the study, and can take responsibility for the integrity of the data and the accuracy of the data analysis. The corresponding author attests that all listed authors meet authorship criteria and that no others meeting the criteria have been omitted.

Conflict of interest: None declared.

Support statement: This work was funded by the Michael Smith Foundation for Health Research, the TB Vets charitable foundation, the Vancouver Coastal Health Research Institute and the University of British Columbia's Strategic Investment Fund. The funding sources had no role in the study design, data collection, data analysis, data interpretation, or writing of the report. Funding information for this article has been deposited with the Crossref Funder Registry.

References

1 World Health Organization. WHO Coronavirus Disease (COVID-19) Dashboard 2021. https://covid19.who.int/ Date last accessed: April 6, 2021. Date last updated: April 6, 2021.

2 Carfi A, Bernabei R, Landi F, et al. Persistent symptoms in patients after acute COVID-19. JAMA 2020; 324: 603-605.

3 Huang Y, Tan C, Wu J, et al. Impact of coronavirus disease 2019 on pulmonary function in early convalescence phase. Respir Res 2020; 21: 163.

4 Zhao Y-M, Shang Y-M, Song W-B, et al. Follow-up study of the pulmonary function and related physiological characteristics of COVID-19 survivors three months after recovery. EClinicalMedicine 2020; 25: 100463.

5 Shah AS, Wong AW, Hague CJ, et al. A prospective study of 12-week respiratory outcomes in COVID-19-related hospitalisations. Thorax 2021: 76: 402-404.

6 Wong AW, Shah AS, Johnston JC, et al. Patient-reported outcome measures after COVID-19: a prospective cohort study. Eur Respir J 2020; 56: 2003276.

7 BC Center for Disease Control. BC COVID-19 Dashboard. www.bccdc.ca/health-info/diseases-conditions/ covid-19/data\#COVID-19Dashboard Date last accessed: April 6, 2021. Date last updated: April 1, 2021.

8 Miller MR, Crapo R, Hankinson J, et al. General considerations for lung function testing. Eur Respir J 2005; 26: 153-161. 
9 Graham BL, Steenbruggen I, Miller MR, et al. Standardization of Spirometry 2019 Update. An Official American Thoracic Society and European Respiratory Society Technical Statement. Am J Respir Crit Care Med 2019; 200: e70-e88.

10 Wanger J, Clausen JL, Coates A, et al. Standardisation of the measurement of lung volumes. Eur Respir J 2005; 26: 511-522.

11 Graham BL, Brusasco V, Burgos F, et al. 2017 ERS/ATS standards for single-breath carbon monoxide uptake in the lung. Eur Respir J 2017; 49: 1600016.

12 Quinones MA, Otto CM, Stoddard M, et al. Recommendations for quantification of Doppler echocardiography: a report from the Doppler Quantification Task Force of the Nomenclature and Standards Committee of the American Society of Echocardiography. J Am Soc Echocardiogr 2002; 15: 167-184.

13 Lang RM, Badano LP, Mor-Avi V, et al. Recommendations for cardiac chamber quantification by echocardiography in adults: an update from the American Society of Echocardiography and the European Association of Cardiovascular Imaging. J Am Soc Echocardiogr 2015; 28: 1-39.e14.

14 Ichikado K, Johkoh T, Ikezoe J, et al. Acute interstitial pneumonia: high-resolution CT findings correlated with pathology. Am J Roentgenol 1997; 168: 333-338.

15 Ichikado K, Suga M, Muller NL, et al. Acute interstitial pneumonia: comparison of high-resolution computed tomography findings between survivors and nonsurvivors. Am J Respir Crit Care Med 2002; 165: 1551-1556.

16 Hatabu H, Hunninghake GM, Lynch DA. Interstitial lung abnormality: recognition and perspectives. Radiology 2019; 291: 1-3.

17 Canadian Institute for Health Information. Patient-reported Outcome Measures (PROMs). www.cihi.ca/en/ patient-reported-outcome-measures-proms Date last accessed: 26 February 2021.

18 Buysse DJ, Reynolds CF, 3rd, Monk TH, et al. The Pittsburgh Sleep Quality Index: a new instrument for psychiatric practice and research. Psychiatry Res 1989; 28: 193-213.

19 Janssen B, Szende A. Population Norms for the EQ-5D. In: Szende A, Janssen B, Cabases J, eds. Self-Reported Population Health: An International Perspective Based on EQ-5D. Dordrecht, the Netherlands, Springer, 2014; pp. 19-30.

20 Kroenke K, Spitzer RL, Williams JB. The PHQ-9: validity of a brief depression severity measure. J Gen Intern Med 2001; 16: 606-613.

21 Eakin EG, Resnikoff PM, Prewitt LM, et al. Validation of a new dyspnea measure: the UCSD Shortness of Breath Questionnaire. University of California, San Diego. Chest 1998; 113: 619-624.

22 Decalmer SC, Webster D, Kelsall AA, et al. Chronic cough: how do cough reflex sensitivity and subjective assessments correlate with objective cough counts during ambulatory monitoring? Thorax 2007; 62: 329-334.

23 Birring SS, Parker D, Brightling CE, et al. Induced sputum inflammatory mediator concentrations in chronic cough. Am J Respir Crit Care Med 2004; 169: 15-19.

24 Lee KK, Matos S, Evans DH, et al. A longitudinal assessment of acute cough. Am J Respir Crit Care Med 2013; 187: 991-997.

25 Kupferberg DH, Kaplan RM, Slymen DJ, et al. Minimal clinically important difference for the UCSD Shortness of Breath Questionnaire. J Cardiopulm Rehabil 2005; 25: 370-377.

26 de Graaf MA, Antoni ML, Ter Kuile MM, et al. Short-term outpatient follow-up of COVID-19 patients: a multidisciplinary approach. EClinicalMedicine 2021; 32: 100731.

27 Bakker C, van der Linden S. Health related utility measurement: an introduction. J Rheumatol 1995; 22: 1197-1199.

28 Ackermann M, Verleden SE, Kuehnel M, et al. Pulmonary vascular endothelialitis, thrombosis, and angiogenesis in Covid-19. N Engl J Med 2020; 383: 120-128.

29 Guler SA, Ebner L, Beigelman C, et al. Pulmonary function and radiological features four months after COVID-19: first results from the national prospective observational Swiss COVID-19 lung study. Eur Respir J 2021; 57: 2003690.

30 Huang C, Huang L, Wang Y, et al. 6-month consequences of COVID-19 in patients discharged from hospital: a cohort study. Lancet 2021; 397: 220-232.

31 Mahler DA, Wells CK. Evaluation of clinical methods for rating dyspnea. Chest 1988; 93: 580-586.

32 Bestall JC, Paul EA, Garrod R, et al. Usefulness of the Medical Research Council (MRC) dyspnoea scale as a measure of disability in patients with chronic obstructive pulmonary disease. Thorax 1999; 54: 581-586.

33 Mahler DA, Ward J, Waterman LA, et al. Patient-reported dyspnea in COPD reliability and association with stage of disease. Chest 2009; 136: 1473-1479. 